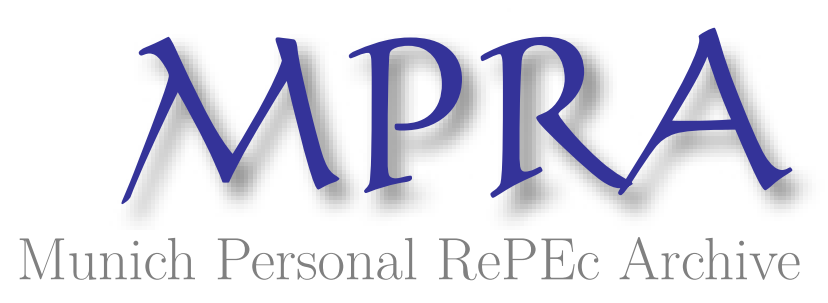

\title{
Absolute vs. Relative Notion of Wealth Changes
}

Kontek, Krzysztof

Artal Investments

16 September 2009

Online at https://mpra.ub.uni-muenchen.de/17336/

MPRA Paper No. 17336, posted 16 Sep 2009 19:41 UTC 


\title{
Absolute vs. Relative Notion of Wealth Changes
}

\author{
Krzysztof Kontek ${ }^{1}$ \\ Artal Investments sp. z o.o. \\ ul. Chrościckiego 93/105, \\ 02-414 Warsaw, Poland \\ e-mail:kontek@artal.com.pl, \\ kkontek2000@yahoo.com \\ tel.:+48 504161750
}

\begin{abstract}
This paper discusses solutions derived from lottery experiments using two alternative assumptions: that people perceive wealth changes as absolute amounts of money; and that people consider wealth changes as a proportion of some reference value dependant on the context of the problem under consideration. The former assumption leads to the design of Prospect Theory, the latter - to a solution closely resembling the utility function hypothesized by Markowitz (1952). This paper presents several crucial arguments for the latter approach and provides strong arguments for rejecting the Prospect Theory paradigm.
\end{abstract}

JEL Classification: C91, D03, D81, D87.

Keywords: Prospect / Cumulative Prospect Theory, Probability Weighting Function, Markowitz Hypothesis, Relative Utility Function, Weber's Law, Decision Making Under Risk.

\section{Introduction}

1.1. Prospect Theory (Kahneman, Tversky, 1979), and its Cumulative version (Tversky, Kahneman, 1992), which describes decision making under conditions of risk and uncertainty, is an alternative to Expected Utility Theory, a hypothesis which has dominated mainstream economic thought since the middle of the twentieth century. Prospect Theory asserts that people are more concerned with changes in wealth than its overall value when making decisions involving small sums of money. Anticipated gains and losses, expressed as mone-

\footnotetext{
${ }^{1}$ I would like to thank Prof. Harry Markowitz for his helpful and valuable comments expressed in private correspondence and during our almost day long meeting on August 14, 2009 in San Diego, CA.
} 
tary amounts, are then used to evaluate the prospects under consideration. However, a probability weighting function is additionally required for this purpose.

The present paper questions the assumption that people treat gains and losses as absolute values when making decisions under conditions of risk. On the contrary, it asserts that gains and losses are perceived in a context which is determined by the way in which the problem is framed and which depends on how attention is focused on it. The result is that gains and losses are perceived in relation to a reference value which, most frequently, is the maximum prospect outcome.

1.2. The paper discusses solutions derived from lottery experiments using two alternative assumptions: that people perceive wealth changes as absolute amounts of money; and that people consider wealth changes as a proportion of the maximum lottery outcome. The former assumption leads to the design of Prospect Theory, the latter to a solution closely resembling the utility function hypothesized by Markowitz (1952).

This paper presents several important arguments in support of the latter approach, the major one being that people regard changes in wealth in a relative way is founded on Weber's Law - one of the fundamental laws of psychophysics. This law contradicts the absolute notion of wealth changes. This observation has also been confirmed by modern researchers including Kahneman, Tversky (1984) and Thaler $(1985,1999)$ since the introduction of Prospect Theory.

Second, an analysis of lottery experiments assuming the absolute notion of wealth changes requires the concept of probability weighting to be incorporated into the descriptive model. Analyzing gains and losses in a relative way, however, eliminates the need for the probability distortion concept.

Finally, the absolute notion of gains and losses leads to ambiguous solutions. Many theories, each proposing a completely different explanation of people's behavior, may be derived from the same set of experimental data. As a result, two contradictory explanations, one claiming that people are generally risk seeking, the other that they are generally risk averse, are sometimes even equally likely when deriving the solution using the probability weighting concept.

1.3. The remainder of the paper is organized as follows. Point 2 shows that treating gains and losses as absolute monetary amounts requires the concept of probability weighting to describe the experimental results. Moreover, it leads to multiple theories being derived from the same set of experimental data. Point 3 presents fundamental observations attesting the perception of gains and losses in context rather than in an absolute way. This allows the 
experimental data to be explained without recourse to a probability weighting function. Such an explanation is presented in Point 4 . Point 5 presents a relative utility function which can be hypothesized using the results presented in Point 4. Point 6 summarizes the article.

\section{Absolute Notion of Wealth Changes}

2.1. Consider the following set of experiments ${ }^{2}$ :

Experiment 1: Would you prefer to enter a lottery with a 50\% chance of winning either $\$ 100$ or $\$ 0$, or to receive a payment of $\$ 50$ ? Most respondents would prefer to receive the certain payment.

Experiment 2: Would you prefer to enter a lottery with a 50\% chance of winning either $\$ 200$ or $\$ 0$, or to receive a payment of $\$ 100$ ? Most respondents would prefer the certain payment.

Experiment 3: Would you prefer to enter a lottery with a 50\% chance of winning either $\$ 400$ or $\$ 0$, or to receive a payment of $\$ 200$ ? Most respondents would prefer the certain payment.

Analogous experiments can be repeated for other monetary amounts in order to "scan" a hypothetical utility function over a broad range of outcomes. Because the responses invariably indicate a preference for certain payments, researchers conclude that people are averse to risk. This is why their utility curve is assumed to be concave whatever the outcome under consideration.

2.2. Consider the following further set of experiments:

Experiment 4: Would you prefer to enter a lottery with a $10 \%$ chance of winning $\$ 500$ and $\$ 0$ otherwise, or to receive a payment of \$50? Most respondents would prefer the lottery.

Experiment 5: Would you prefer to enter a lottery with a 10\% chance of winning $\$ 1000$ and $\$ 0$ otherwise, or to receive a payment of $\$ 100$ ? Most respondents would prefer the lottery.

Experiment 6: Would you prefer to enter a lottery with a $10 \%$ chance of winning $\$ 2000$ and $\$ 0$ otherwise, or to receive a payment of $\$ 200$ ? Most respondents would prefer the lottery.

The respondents in these cases exhibit risk seeking behavior and, interestingly, for the same certain payment amounts $(\$ 50, \$ 100$, and $\$ 200)$ as in Experiments 1 - 3. Researchers

\footnotetext{
${ }^{2}$ These are mental experiments only. However similar experiments have been conducted by numerous authors including Kahneman, Tversky $(1979,1992)$, and Gonzales, Wu (1999). CPT's parameters have been estimated basing on the results of two-outcome lotteries. Gonzales and $\mathrm{Wu}$ proceeded in a similar way.
} 
conclude that people are generally risk averse (as demonstrated in Experiments $1-3$ ) in these situations as well, but that there has to exist an additional effect related to the perception of probabilities in order to explain the risk-seeking behavior observed in Experiments 4 - 6. This effect would have to rely on a non-linear perception of probabilities and especially on an overweighting of low ones. This reasoning thus leads to a theory in which there exists a nonlinear probability weighting function in addition to a utility function. This is how Prospect Theory (and other theories using a similar approach) developed.

2.3. There is so far no reason to reject this solution. However, there is no reason why these experiments could not have been conducted in the reverse order to arrive at the very opposite conclusions. Researchers could well have concluded that people are generally riskseeking on the strength of Experiments $4-6$ and argued that their utility function should be convex over the entire range of outcomes under consideration. The risk-aversion observed in Experiments $1-3$ would again find its explanation in a non-linear probability weighting, only this time the low probabilities would be perceived accurately whereas probabilities around 0.5 would be heavily underweighted.

Such an explanation would also utilize both the utility and probability weighting functions. But a very different theory would result with both curves differing in shape from those in the first theory. Both theories would have the same ability to describe (or rather to fit) the experimental results, albeit with completely different modi operandi. The fact that changing the sequence of reasoning steps changes the resulting explanation is then a very serious signal that this approach is unsound.

2.4. Moreover, the researchers could have started with yet another probability of winning the main prize somewhere between 0.1 and 0.5 . Having conducted a series of experiments, they would have devised another theory, this time stating that people are generally risk neutral over the broad range of outcomes. This theory would, however, require yet another shape of the probability weighting function in order to fit the experimental results.

This shows that an unlimited number of such theories could be derived from the same set of experimental data. As a result, we cannot vouch for the correctness of any conclusion arrived at by utilizing this line of reasoning ${ }^{3}$.

\footnotetext{
${ }^{3}$ Gonzales and Wu (1999) offer the best example of this. Their "On the Shape of the Probability Weighting Function" paper is commonly regarded as one of the major contributions to Prospect Theory and is therefore frequently cited in the literature on the subject. This is due to the fact that it provides a deeper insight into the psychology of the probability weighting function while discussing several of its characteristics such as curvature (which is explained using the discriminability concept met in the psychophysics literature) and elevation (which is interpreted as the attractiveness of a game to a particular subject). The authors go so far as to state that: "there appear to be two logically independent psychological properties that characterize the weighting function". The
} 


\section{Relative Notion of Wealth Changes}

3.1. The foregoing reasoning assumed the absolute notion of wealth changes. This means that gains and losses were represented and analyzed as monetary amounts (such as $\$ 50$, $\$ 100$, or $\$ 200$ ). This is one of the basic assumptions of Prospect Theory and is best expressed by a value function which supposedly determines the value (utility) of specific amounts of money to people ${ }^{4}$.

People, however, typically consider wealth changes in a relative way. This means that gains and losses are usually perceived as a proportion of a reference value which depends on the context of the problem ${ }^{5}$. This observation is not new and was also noticed by Kahneman and Tversky in 1984, i.e. 5 years after the introduction of Prospect Theory ${ }^{6}$. Despite this, the absolute notion of gains and losses remained the underlying assumption of Cumulative Prospect Theory, which appeared in 1992.

3.2. The explanation that people regard changes of wealth in a relative way is founded on basic psychophysical laws. In the first half of the nineteenth century, German researcher Ernst Weber conducted experiments on determining the Just Noticeable Difference in weight between objects and concluded that this difference is twice as great with a $2 \mathrm{~kg}$ object than with a $1 \mathrm{~kg}$ object. The law Weber formulated in 1834 states that this difference is a constant

most interesting statement is however to be found in Footnote 11: "There was a high multicolinearity between the elevation of the probability weighting function ... and the exponent of the value function. .... These two parameters correlated -0.98 .... The curvature parameter for the probability weighting function and the exponent of the value function ...correlated ... -0.66. The two-parameter Prelec function also exhibited high multicolinearity between the elevation parameter and the a parameter (0.97)." This high multicolinearity simply means that the value and probability weighting functions are very strongly interdependent and they contain pretty much the same information about the phenomena described. The model is not statistically robust as a result. This can be avoided by dropping some of the descriptive variables. In this case, however, it may mean dropping one of the descriptive functions. Additionally, none of the "psychological" considerations regarding the shape of the value and probability weighting functions appear to have any special significance.

${ }^{4}$ According to Cumulative Prospect Theory the value function is defined as $v(x)=\lambda|x|^{\alpha}$, where $x$ is the gain or loss expressed as an absolute monetary amount.

${ }^{5}$ To make it absolutely clear: Prospect Theory asserts that people consider changes in wealth rather than its overall value and introduces the concept of gains and losses expressed as absolute amounts of money. This may be referred to as the "absolute notion of wealth changes". It is additionally assumed in this paper that those wealth changes (e.g. gains and losses) are perceived as proportions (percentages) of a reference value determined by the context of the problem under consideration. This is referred to as the "relative notion of wealth changes".

${ }^{6}$ Kahneman and Tversky consider a minimal, topical, and comprehensive account in their "Choices, Values, and Frames" paper. They state: A topical account relates the consequences of possible choices to a reference level that is determined by the context within which the decision arise and conclude: People will spontaneously frame decisions in terms of topical accounts. ... The topical organization of mental accounts leads people to evaluate gains and losses in relative rather than in absolute terms (emphasis added). The idea of mental accounts actually originated with Thaler $(1985,1999)$. 
proportion of the initial stimulus magnitude ${ }^{7}$. This is now one of the fundamental laws of psychophysics (Encyclopædia Britannica, 2009):

$$
\Delta S / S=k
$$

where $\Delta S$ is the Just Noticeable Difference, $S$ is the initial stimulus magnitude and $k$ is a constant. This law holds with reasonable accuracy for most stimuli within a broad range. For example, the value of $k$ is $2 \%$ for weight, $4.8 \%$ for loudness and $7.9 \%$ for brightness. It follows from the Weber law that the same change in stimulus (for instance $0.2 \mathrm{~kg}$ ) can be strongly felt, slightly noticed or not perceived at all depending on the magnitude of the initial stimulus. It further follows that an unambiguous and absolute perception level of a specific stimulus change cannot be determined, as this depends on the situational context.

3.3. How the Weber Law works for financial stimuli will be presented with the following example:

Problem 1a: What is the smallest significant amount of money to a person shopping for goods worth about \$100?

This will possibly be close to 1 dollar (but not as small as 1 cent). Such a person may, for example, consider choosing a rival product that is $\$ 0.5$ cheaper.

Problem 1b: What is the smallest amount of money considered by the same person purchasing a house for $\$ 500,000$ ?

This will probably be at least $\$ 1,000$. A purchase offer of $\$ 479,538$ is hard to imagine; $\$ 480,000$ seems far more likely. It follows that one dollar, a significant amount in the former case, is completely insignificant in the latter. Even $\$ 100$, the sum total of a person's expenditure in a shop, is of no significance in a house purchase.

3.4. The foregoing example demonstrates that the human mental system adapts itself to financial quantities, just as its sensory system does to physical ones. The result is that the Just Noticeable Difference remains an approximately constant proportion of different financial amounts. This means that when considering financial prospects (projects, investments, lotteries etc.), the size of the prospect becomes a reference value in the entire mental process, rendering an absolute amount of money (say \$10) relevant or irrelevant depending on the context. This conclusion constitutes a fundamental deviation from Prospect Theory, which regards gains and losses in absolute terms, and attempts to derive a value function in terms of absolute monetary amounts ${ }^{8}$.

\footnotetext{
${ }^{7}$ An extension of the Weber Law is the Weber-Fechner Law, which was formulated in 1860 and describes the logarithmic perception of stimuli.

${ }^{8}$ This does not even seem possible according to the Weber law.
} 


\section{Solution Resulting From Relative Notion of Wealth Changes}

4.1. In point 2, we demonstrated how the consideration of gains and losses in absolute terms inevitably leads to the concept of probability weighting. We further demonstrated that this approach produces ambiguous results. Many theories can be derived from the same set of experimental data, each theory having the same ability to describe (fit) the experimental results. We will now show that considering gains and losses as relative values allows the experimental results to be explained without the probability weighting concept and leads to a single, unambiguous solution.

4.2. The results of the Experiments 1-6 can be explained differently by assuming that people perceive outcomes as proportions of the main payment. No "scanning" of the utility function is performed for the various outcomes in Experiments 1 - 3 because the amount of the certain payment is always half that of the main payment. All three questions therefore deal with the value of the utility function for the relative outcome of $r=0.5$. The conclusion from these three experiments is that people are risk-averse for this particular relative outcome, so the utility function is concave at this point. But this conclusion only concerns this one point. Repeating similar experiments for different outcome values would still only inform us about this single point of the curve expressed in terms of relative outcomes.

Similarly, Experiments 4 - 6 show that people exhibit a risk-seeking attitude by their preference for the lottery over a relative outcome of $r=0.1$. The utility function is therefore convex at this point. Once again, this conclusion is only valid for this single point. This line of reasoning leads to the plotting of a utility function expressed for relative outcome values, which is partially convex and partially concave. This would certainly be a completely different solution from that proposed by Prospect Theory, all the more so since it would not utilize any probability weighting function.

4.3. The foregoing reasoning may be repeated for losses as well. Consider the following set of 3 experiments (the outcomes in parentheses correspond with the respective experiment numbers):

Experiment $7(8,9)$ : Would you prefer to enter a lottery with a 90\% chance of losing $\$ 100$ (\$200, \$400) and \$0 otherwise, or to pay $\$ 90(\$ 180, \$ 360)$ to avoid the game? Most respondents would prefer the lottery.

Consider now the following further set of 3 experiments: 
Experiment $10(11,12)$ : Would you prefer to enter a lottery with a 10\% chance of losing $\$ 900$ (\$1800, \$3600) and $\$ 0$ otherwise, or to pay $\$ 90(\$ 180, \$ 360)$ to avoid the game? Most respondents would prefer to pay the certain amount.

The respondents in Experiments 7 - 9 exhibit risk seeking behavior, whereas the respondents in Experiments 10 - 12 exhibit risk aversion behavior for exactly the same certain payment amounts $(\$ 90, \$ 180$, and $\$ 360)$. As in case of gains, considering losses in absolute terms requires a probability weighting function to be incorporated into the descriptive model. Similarly, the order of the reasoning steps influences the conclusion regarding the general attitude to risk. Beginning with Experiments 7 - 9 leads to the conclusion that people are generally risk seeking and that a probability weighting function is required to explain the risk aversion observed in Experiments 10 - 12. Conversely, beginning with Experiments 10 - 12 leads to the conclusion that people are generally risk averse and that a probability weighting function is required to explain the risk seeking attitude observed in Experiments 7 - 9.

Once again, it follows that completely different modi operandi result from both approaches with the value and probability weighting functions assuming different shapes in each case. Which solution truly explains the behavior of the respondents is therefore a fair question. The situation becomes even more embarrassing once we notice that the probabilities $10 \%$ and $90 \%$ are symmetrically located on the probability scale and that neither should be preferred over the other when choosing the sequence of reasoning steps. We are therefore inexorably drawn to the paradoxical conclusion that both solutions (one claiming that people are generally risk seeking, another that they are generally risk averse) are equally likely on the basis of Experiments 7 - 12.

4.4. Analyzing the experimental results using the relative notion of losses leads to a completely different and unambiguous result. Experiments 7 - 9 lead to the conclusion that people are risk seeking for the relative loss of $r=0.9$, whereas Experiments $10-12$ lead to the conclusion that people are risk averse for the relative loss of $r=0.1$. No probability weighting function is required to explain the results of these experiments.

\section{Relative Utility Function}

5.1. The shape of the utility function, expressed in terms of relative outcomes, can now be hypothesized using the results presented in Point 4. The solution is presented in Fig.1. 


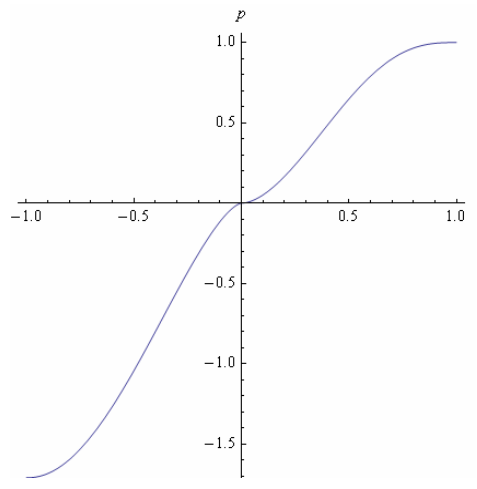

Fig. 1. The relative utility function.

The curve should be concave for high relative gains, reflecting the risk aversion observed in Experiments 1-3, and convex for low relative gains, reflecting the risk seeking attitude observed in Experiments 4-6.

The picture is reversed for losses. The function should be convex for high relative losses, reflecting the risk seeking attitude observed in Experiments 7 - 9, and concave for low relative losses, reflecting the risk aversion observed in Experiments $10-12$.

The double-S shape of this hypothetical relative utility function offers a simple and concise explanation of risk-seeking and risk-aversion attitudes when making decisions under conditions of risk. This replaces the "fourfold pattern" of risk attitude formulated by Cumulative Prospect Theory. This solution is unambiguous and does not require a probability weighting function to describe the pattern.

The curve should be of greater magnitude for losses than for gains as people are generally averse to loss.

5.2. Quite surprisingly, the obtained curve strongly resembles the utility function hypothesized by Markowitz (1952) and shown in Figure 2.

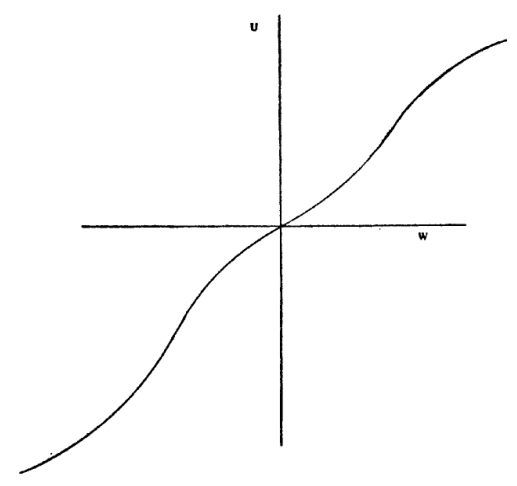

Fig. 2. The shape of the utility function according to the Markowitz hypothesis of 1952. 
The only significant difference with the Markowitz curve is that the relative utility function is defined for relative values of outcomes rather than for absolute ones.

\section{Summary.}

This paper discusses solutions derived from lottery experiments using two alternative assumptions: the absolute and relative notion of wealth changes. Several important advantages of the latter approach have been presented in the paper. Most importantly, people do consider changes of wealth in a relative way. This was even confirmed by Kahneman and Tversky - the authors of Prospect Theory.

However, introducing this assumption into an analysis of the experimental data leads to the rejection of Prospect Theory itself. The concept of probability weighting - one of the key planks of Prospect Theory - becomes unnecessary when gains and losses are considered in a relative way.

It was shown that retaining the concept of probability weighting leads to multiple solutions being derived from the same set of experimental data. A paradox was presented that two contradictory explanations - one claiming that people are generally risk seeking, the other that they are generally risk averse - might even be equally likely if probability weighting is utilized.

The relative notion of wealth changes leads to a completely different and unambiguous solution, one which strongly resembles the utility function hypothesized by Markowitz (1952).

This paper strictly demarcates the two approaches as the relative and absolute notions are mutually exclusive. As a result Prospect Theory cannot be held to be an accurate explanation of people's behavior while gains and losses are simultaneously held to be perceived in a relative way.

\section{References}

Gonzales, R., Wu, G., (1999). On the Shape of the Probability Weighting Function. Cognitive Psychology, 38, pp 129-166.

Kahneman, D., Tversky, A., (1979). Prospect theory: An analysis of decisions under risk. Econometrica, 47, pp 313-327.

Kahneman, D., Tversky, A., (1984). Choices, Values, and Frames. American Psychologist, Vol. 39, No. 4, pp 341-350.

Markowitz, H., (1952). The Utility of Wealth. Journal of Political Economy, Vol. 60, pp 151-158. 
Thaler, R. H., (1985). Mental accounting and consumer choice. Marketing Science, 4, pp 199-214.

Thaler, R. H., (1999). Mental Accounting Matters. Journal of Behavioral Decision Making, Vol.12, pp 183-206.

Tversky, A., Kahneman, D., (1992). Advances in Prospect Theory: Cumulative Representation of Uncertainty. Journal of Risk and Uncertainty, Springer, vol. 5(4), pp 297-323.

Weber's law. (2009). In Encyclopcedia Britannica. Retrieved August 11, 2009, from Encyclopædia Britannica Online: http://www.britannica.com/EBchecked/topic/638610/Webers-law 